\title{
Chilaiditi syndrome
}

\author{
Ozge Karaman, Muzaffer Kahyaoglu, Emine Alpay, Arzu Kalaycı, and Cetin Gecmen
}

Department of Cardiology, Kartal Kosuyolu Heart \& Research Hospital, Istanbul, Turkey

Received: December 3, 2016

Revised : January 19, 2017

Accepted: July 15, 2017

\section{Correspondence to} Muzaffer Kahyaoglu, M.D.

Tel: +90-5062333599

Fax: $+90-2165001500$

E-mail: mkahyaogluo9@hotmail.com

A 6o-year-old male patient was referred to our clinic because of persistent hiccups and epigastric pain. His past medical history was unremarkable for any chronic illness. On physical examination, there was tenderness in the right upper quadrant and epigastric area. Bowel sounds were normal and there was no rebound tenderness and guarding. His blood work including electrolytes, complete blood count and hepatic panel were with in normal limits. Chest radiography revealed that the right hemidiaphragm was elevated (Fig. 1). Abdominal computed tomography scan demonstrated that a loop of bowel interposed between the liver and diaphragm in axial view (Fig. 2). The patient was diagnosed with Chilaiditi syndrome. High fiber diet and laxatives were recommended the patient. After treatment, his compliants were

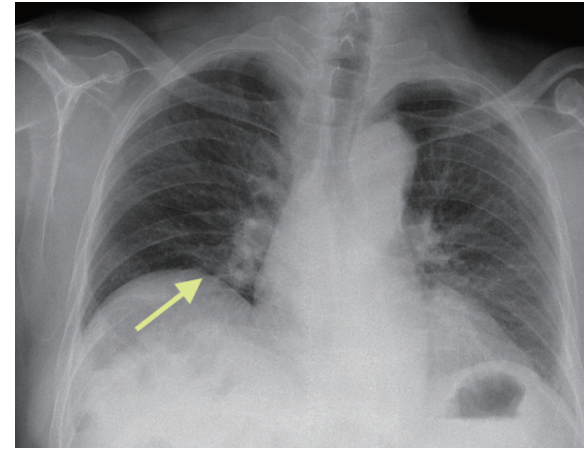

Figure 1. Chest radiography shows elevation of right hemidiaphragm (arrow). resolved.

Chilaiditi syndrome is extremely rare entity. This entity was first described by Demetrius Chilaiditi in 1910. Chilaiditi's sign is defined as colon or small intestine interposition between liver and diaphragma. When this sign associated with gastrointestinal symptoms, this entity is known as Chilaiditi syndrome. The treatment of Chilaiditi syndrome is usually nonsurgical and includes bed rest, fluid supplementation, nasogastric decompression, enemas, cathartics, a high-fiber diet, and stool softeners. Thus, we want share the demonstrative views of this rare entity.

\section{Conflict of interest}

No potential conflict of interest relevant to this article was reported.

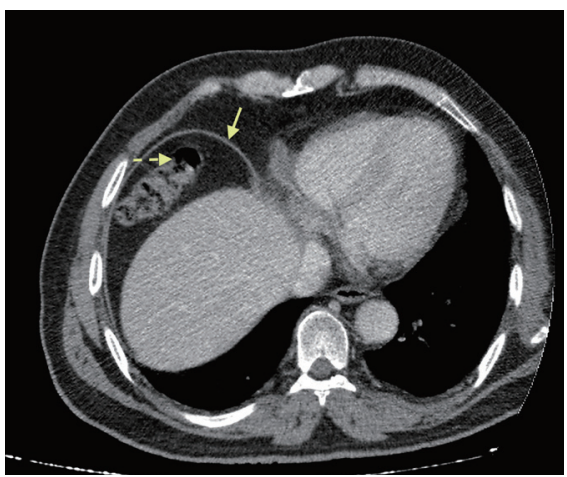

Figure 2. Abdominal computed tomography shows interposition of bowel (dotted arrow) between liver and right hemidiaphragm (arrow) in axial view. 